\title{
Sonographic Assessment of Myometrial Thickness as A Predictor for the Latency Interval in Women with Preterm Premature Rupture of Membranes
}

\author{
Abdalmonem Mohamed Zakaria, Waleed Alsayed Alsayed Alnagar, Khaled Mahmoud Ahmed Ahmed \\ Department of Obstetrics and Gynecology, Faculty of Medicine, Al-Azhar University, Cairo, Egypt \\ Corresponding author: Khaled Mahmoud Ahmed Ahmed, Mobile: (+20)01007665054, E-Mail: dr.khaled.m.a.a@gmail.com
}

\begin{abstract}
Background: Spontaneous rupture of membranes is a normal component of labor and delivery. Rupture of membranes before the onset of labor is considered premature (PROM), and induction of labor is common if the patient is at or close to term. Patient management becomes more challenging when rupture of membranes occurs preterm (PPROM), and in the absence of labor.

Objective: The aim of this work was to measure the myometrial wall thickness (MT) by ultrasound scanning in women with PPROM as predictors for the latency interval, and see other impacts that predict the latency interval. Patients and Methods: This study included a total of 100 pregnant women attending at Labor and Delivery Ward in Al Hussein Hospital, Al-Azhar University. This study was conducted between May 2018 to December 2018. Pregnant women were divided into three groups: preterm premature rupture of membranes (PPROM, $\mathrm{n}=50$ ), and term nonlabor control (T-CTR, $\mathrm{n}=25$ ) and preterm nonlabor control (P-CTR, $\mathrm{n}=25)$.

Results: revealed that women with PPROM were slightly older compared with those in P-CTR group and TCTR group but with no statistical significant difference. There was no significant difference among groups in number of previous pregnancies. There was also no significant difference in maternal body weight among groups. Regression analysis suggested that there was a direct correlation between latency interval and fundal MT with a very strong positive Pearson correlation and a highly significant $\mathrm{p}$ value.

Conclusion: It could be concluded that there was a significant thickening of the fundal walls of the uterus follow PPROM. A thick myometrium in no laboring PPROM women is associated with long latency interval.

Keywords: PPROM, Myometrium, Oligohydramnios Ultrasound preterm birth, latency interval

\section{INTRODUCTION}

Spontaneous rupture of membranes is a normal component of labor and delivery. Rupture of membranes before the onset of labor is considered premature (PROM), and induction of labor is common if the patient is at or close to term. Patient management always becomes more challenging when rupture of membranes occurs preterm (PPROM), and in the absence of labor. The natural history of PROM progresses in such a way that $90 \%$ of term patients and $50 \%$ of preterm patients enter spontaneous labor within 24 hours. The major question regarding management of these patients is whether to allow them to enter labor spontaneously or to induce labor. In large part, the management of these patients depends on their desires ${ }^{(\mathbf{1})}$.

Many cases of PPROM are caused by idiopathic weakening of the membranes ${ }^{(2)}$. Other causes include incompetent cervix, abruption placenta, and amniocentesis. The incidence of PPROM ranges from $2 \%$ to $20 \%$ and is associated with $18 \%$ to $20 \%$ of perinatal deaths ${ }^{(3)}$.

Perinatal risks with PPROM are primarily complications from immaturity including respiratory distress syndrome, intraventricular Hemorrhage, patent ductus arteriosus, and necrotizing enterocolitis ${ }^{(4)}$.

Pulmonary hypoplasia is the most serious complications, and can be lethal, the presence of severe (AFI less than $2 \mathrm{~cm}$ ), prolonged (more than

14 days), and early (less than 25 weeks at onset) oligohydramnios has been associated with a neonatal mortality rate greater than $90 \%{ }^{(5)}$.

Similar to myocardium, the force of labor is uterine wall tension opposed to the resistance of the cervix, perineum and pelvis. Mathematical modeling reveals that uterine wall stress (defined as applied force per unit cross- sectional area of material) is directly proportional to both the intracavitary pressure and the radius of the curvature, but inversely proportional to the thickness of the myometrium. Thus, the thicker the myometrium, the lower the uterine wall stresses ${ }^{(6)}$.

Sonographic observation that the myometrium thins symmetrically during active labor with the least amount of thinning at the uterine fundus stimulate scientists to rethink the mechanisms responsible for the uniform dispersion of the contractile forces that insure efficient fetal expulsion. Sudden decompression with the uterine sac, which has been filled with a minimally compressible fluid that normally opposed thickening, is the most likely physiologic explanation ${ }^{(7)}$.

A direct correlation was found between fundal myometrial thickness (MT) and the latency interval (LI). Showing that the thicker the fundal myometrium, the longer the latency interval ${ }^{(8)}$.

The latency interval is defined as the time period (days or hours) from the time of rupture of membranes reported by the patients to delivery. Our
\end{abstract}


understanding of the mechanisms that determined the length of the latency interval after PPROM is hindered by the fact that the human myometrium and cervix appear to have redundant and parallel mechanisms to ensure adequate length of gestation (8). Furthermore the impact of pregnancy and labor on the uterus and cervix differs greatly ${ }^{(9)}$.

The prevailing theories surrounding PPROM latency interval may overestimate the importance of the cervix, leaving the role played by myometrial activation largely unexplored (10). The digital cervical examination and frequency of uterine contractions have weak prognostic values ${ }^{(\mathbf{1 1})}$. Not only are digital cervical examination of women with PPROM and frequency of uterine contractions poorly predictive, but a digital exam may actually increase the risk of ascending infection.

There has been much attention focused on the sonographic assessment of cervical length since shortening is associated with an increased risk of preterm delivery in both nulliparous and multiparous women ${ }^{(\mathbf{1 2})}$. Sonographic evaluation of cervical length in women with PPROM is reported to have maximum sensitivities and specificities of $63 \%$ and $81 \%$ respectively ${ }^{(13)}$.

It is assumed that women with a long latency interval after spontaneous PPROM are in a state of myometrial quiescence or incomplete myometrial activation, and it is demonstrated that the long latency and presumed myometrial quiescence are associated with a greater thickness of the anterior and fundal wall myometrium. It is possible that those women with PPROM and thin myometrium already experienced functional complete myometrial activation that allows for co-ordinate tone, contractions, and shorter latency interval ${ }^{\left({ }^{(8)}\right.}$.

The aim of this work was to measure the myometrial wall thickness (MT) by ultrasound scanning in patients with preterm premature rupture of membranes as a predictor for the latency interval.

\section{PATIENTS AND METHODS}

This study included a total of 100 pregnant women attending at Labor and Delivery Ward in Al Hussein Hospital, Al-Azhar University. Approval of the ethical committee and a written informed consent from all the subjects were obtained. This study was conducted between May 2018 to December 2018.

The pregnant women enrolled in the study were divided into three groups:

- Group I: included 50 women with preterm premature rupture of membranes with gestational age from 24 to 34 weeks.

- Group II: included 25 term non-labor control with gestational age from 37 to 41 weeks.

- Group III: included 25 preterm non-labor control with gestational age from 24 to 34 weeks.

Inclusion criteria: women with singleton pregnancy for the three groups and with a definite history of current PPROM for the study group.

Exclusion criteria for the three groups included:

1- Suspected fetal growth restriction (IUGR).

2- Any gross fetal anomalies.

3- Abnormalities of placentation.

4- Uterine structural abnormalities. (IUFD).

5- Fetal jeopardy or intrauterine fetal death

Exclusion criteria for women with PPROM: All of the above criteria plus specific criteria as:

1. Women presenting with chorioamniointis with fever over $38 \mathrm{c}$ abdominal tenderness, foul vaginal discharge and/or fetal tachycardia

2. Diabetics, immunocompromized and cardiac patients.

3. Women with cervical cerclage.

4. Women with drained liquor.

\section{All pregnant women were subjected to:}

1. History: Full history was taking from all women including personal history for maternal age, obstetric history for number of previous pregnancies, history of previous PPROM. History of present pregnancy included gestational age, history of drug intake during the present pregnancy with special emphasis to women with PPROM giving history of gush of clear watery fluid from the vagina. Past history was useful to exclude women with a contraindication for conservative management in the study group.

2. Physical examinations: General examination was done for the three groups for pulse, arterial blood pressure and temperature to exclude any signs of chorioamnionitis in the study group. Abdominally, fundal level examinations for the three groups were done for predicting the gestational age and/or IUGR or abnormalities of liquor. Monitoring of uterine contraction helped to pick up women who are not in labor in the study group. P/V examination was not done for the three groups.

3. While definite diagnosis of PPROM relied on the presence of a moist perineum, and/or sterile speculum examination showing a collection fluid in the vagina. Some women needed direct observation of the cervix during a valsulva maneuver or with cough to show free flow of fluid from the cervical os. 
4. Ultrasonographic investigation: Ultrasound scanning was performed for the three groups and was performed within 12 hours from onset of PPROM in the study group to avoid drained liquor or development of chorioamnionitis. One investigator conducted all ultrasound examinations. An abdominal ultrasound was performed using a $3.5 \mathrm{MHz}$ transabdominal probe, the procedure was carried while women were in supine position, the transducer was covered by coupling gel and the scanning was done symmetrically starting from the fundus of the uterus for:

a) Estimation of gestational age,

b) Assessment of fetal wellbeing by biophysical profile in the study group.

c) Detection of any fetal gross anomalies.

d) Assessment of fetal growth to exclude IUGR.

e) Detection of any abnormal placental insertion and/or uterine structural abnormalities as septate or bicornuate uterus.

5. The myometrial thickness:

The myometrium was sonographically detected as the echo homogenous layer between the serosa and the decidua. The myometrial thickness was measured at 4 different sites:

a) The lower uterine segment (LUS): LUS was identified as a layer which lies approximately $2 \mathrm{~cm}$ above the reflection of the urinary bladder. The bladder had to be full during scanning for accurate measurement of the LUS ${ }^{(\mathbf{1 4})}$.

b) The anterior wall: The anterior layer was identified with the scan probe $1 \mathrm{~cm}$ above the maternal umbilicus ${ }^{(14)}$.

c) Fundus: The thickness of the fundus was measured by placing the scan probe perpendicularly above the uterine fundus so that the entire curvature of the uterus was visualized.

d) The posterior wall: Measurement of the posterior wall was technically the most challenging. We demarcated the posterior wall using the maternal abdominal aortic pulsation as an anatomic marker. Each measurement was made from separate scan images; at least 3 measurements were obtained at each site and averaged.

\section{Amniotic fluid index (AFI):}

The amniotic fluid volume was measured using the 4-quadrant technique as described by Phelan et al (1987), the abdomen of the pregnant women was divided into 4 quadrants using the umbilicus as a reference point to divide the uterus into upper and lower halves and by using the linea nigra to divide the uterus into right and left halves. Determination of amniotic fluid volume (AFV) was achieved by the calculation of the depth of the largest vertical pocket in each quadrant, the 4 measurements were summed to obtain the amniotic fluid index in centimeters. The ultrasound transducer was held along the maternal longitudinal axis and maintained perpendicular to the floor while measuring the AFI. Pockets should be free from fetal limbs andlor umbilical cord ${ }^{(\mathbf{1 5})}$. As described by Phelan et $\boldsymbol{a l}$. (16), we diagnosed oligohydramnios when AFI was less than $5 \mathrm{~cm}$.

\section{Fetal biometric assessment:}

Sonographic estimation of fetal weight (SEFW) was done by obtaining fetal biometric information to allow for accurate algorithmic calculation of fetal weight.

The Hadlock et al. ${ }^{\left({ }^{17)}\right.}$ formula was chosen for assessment of fetal weight by biometric linear measurement of the abdominal circumference (AC), the biparietal diameter (BPD) and femur length (FL)

\section{Conservative management for women with PPROM consisted of the following:}

- Temperature chart every 6 hours.

- Total leucocytic count (TLC) and Creactive protein (CRP) twice weekly.

- Biophysical profile (BPP) and/or cardiotogography (CTG) twice weekly for assessment of fetal well being and/or uterine contractions for exclusion of developing labor.

- Corticosteroid administration in the form Dexamethazone $12 \mathrm{mg}$ IV/12 hours for two doses.

- Erythromycin 500mg every 6 hours for one week.

- Tocolysis and/or digital examination were not permitted.

\section{Statistical analysis}

Recorded data were analyzed using the statistical package for social sciences, version 20.0 (SPSS Inc., Chicago, Illinois, USA). Quantitative data were expressed as mean \pm standard deviation (SD). Qualitative data were expressed as frequency and percentage.

\section{The following tests were done:}

- Independent-samples t-test of significance was used when comparing between two means.

- Chi-square $\left(\mathrm{x}^{2}\right)$ test of significance was used in order to compare proportions between two qualitative parameters.

- The confidence interval was set to $95 \%$ and the margin of error accepted was set to $5 \%$. The pvalue was considered significant as the following:

- Probability (P-value)

- P-value <0.05 was considered significant. 
- P-value <0.001 was considered as highly significant.

- $\quad$ P-value >0.05 was considered insignificant.

\section{RESULTS}

Demographic variables assessed were:

- Maternal age.

- Number of previous pregnancies.
- Maternal weight.

While clinical and sonographic variables were:

- Gestational age.

- Sonographically estimated fetal weight (SEFW).

- Amniotic fluid index (AFI).

One way AVOVA test was used to determine the $\mathrm{P}$ value.

Table (l): Demographic data in PPROM and T-CTR

\begin{tabular}{|c|c|c|c|c|c|}
\hline & \multirow[t]{2}{*}{ Group } & \multirow[t]{2}{*}{ Mean $\pm S D$} & \multicolumn{2}{|c|}{$95 \%$ Confidence interval CI } & \multirow[t]{2}{*}{$P$} \\
\hline & & & lower bound & upper bound & \\
\hline \multirow[t]{2}{*}{ Age (years) } & PPROM & $29.44 \pm 6.1$ & 27.68 & 31.21 & \multirow[t]{2}{*}{0.57} \\
\hline & T-CTR & $26.76 \pm 5.9$ & 24.32 & 29.20 & \\
\hline \multirow{2}{*}{$\begin{array}{l}\text { Previous } \\
\text { pregnancies }\end{array}$} & PPROM & $2.20 \pm 2.1$ & 1.60 & 2.80 & \multirow[t]{2}{*}{0.61} \\
\hline & T-CTR & $1.68 \pm 1.8$ & 0.92 & 2.44 & \\
\hline \multirow{2}{*}{$\begin{array}{l}\text { Maternal } \\
\text { Weight }(k g)\end{array}$} & PPROM & $85.1 \pm 6.0$ & 83.62 & 88.72 & \multirow[t]{2}{*}{0.23} \\
\hline & T-CTR & $89.8 \pm 4.1$ & 86.56 & 91.43 & \\
\hline
\end{tabular}

Table (2):Demographic data in PPROM and P-CTR

\begin{tabular}{|c|c|c|c|c|c|}
\hline & \multirow[t]{2}{*}{ Group } & \multirow[t]{2}{*}{$\begin{array}{l}\text { Mean } \\
\pm S D\end{array}$} & \multicolumn{2}{|c|}{$\begin{array}{l}\text { 95\% Confidence interval } \\
\text { CI }\end{array}$} & \multirow[t]{2}{*}{$P$} \\
\hline & & & lower bound & upper bound & \\
\hline \multirow[t]{2}{*}{ Age (years) } & PPROM & $29.44 \pm 6.1$ & 27.68 & 31.21 & \multirow[t]{2}{*}{0.54} \\
\hline & P-CTR & $26.56 \pm 5.9$ & 24.72 & 28.40 & \\
\hline \multirow{2}{*}{$\begin{array}{l}\text { Previous } \\
\text { pregnancies }\end{array}$} & PPROM & $2.20 \pm 2.1$ & 1.60 & 2.80 & \multirow[t]{2}{*}{0.57} \\
\hline & P-CTR & $1.6 \pm 2.5$ & 0.64 & 2.72 & \\
\hline \multirow{2}{*}{$\begin{array}{l}\text { Maternal } \\
\text { Weight }(\mathrm{kg})\end{array}$} & PPROM & $85 . \pm 6.0$ & 83.62 & 88.72 & \multirow[t]{2}{*}{0.74} \\
\hline & P-CTR & $82.7 \pm 3.9$ & 81.14 & 84.38 & \\
\hline
\end{tabular}

As shown in tables (1 and 2) women with PPROM were slightly older compared with those in T-CTR groups and P-CT group but the difference was not statistically significant

As shown in tables (1 and 2). There was no significant difference in maternal body weight between PPROM and the two controlled groups (1and 2).

There was no significant difference among groups in number of previous pregnancies as shown in tables

Table (3): The post-hoc Tukey test showing the difference in gestational age between PPROM, P-CTR and T-CTR groups

\begin{tabular}{|l|c|c|c|c|}
\hline \multirow{2}{*}{ Groups } & \multirow{2}{*}{$N$} & \multicolumn{2}{|c|}{ Subset for alpha= 0.05 } \\
\cline { 3 - 5 } & $N$ & 1 & 2 & 3 \\
\hline P-CTR & 25 & 31.04 & & 31.04 \\
\hline T-CTR & 25 & & 39.04 & 39.04 \\
\hline PPROM & 50 & 30.74 & 30.74 & \\
\hline Significance & & 0.884 & 1.000 & 1.000 \\
\hline
\end{tabular}

Regarding the gestational age, women with PPROM (mean \pm SD: $30.7 \pm 2.8 \mathrm{w}$ ) and P-CTR group (mean $\pm \mathrm{SD}: 31 \pm 2.4 \mathrm{w}$ ) showed no significant difference with post-hoc significance $=0.884$ and $\mathrm{p}>0.05$, while there was a significant difference in GA between PPROM and T-CTR group (mean \pm SD: $39 \pm 1.3 \mathrm{w}$ ) with post-hoc significance $=1$ and $\mathrm{p}<0.001$. As well as between P-CTR group and T-CTR group with post-hoc significance $=1$ and $\mathrm{p}<0.001$. (Table 3$)$. 
Table (4): The post-hoc Tukey test showing the difference in fetal weight between PPROM, P-CTR and TCTR groups.

\begin{tabular}{|l|c|c|c|c|}
\hline \multirow{2}{*}{ Groups } & \multirow{2}{*}{$N$} & \multicolumn{3}{|c|}{ Subset for alpha= 0.05} \\
\cline { 3 - 5 } \cline { 3 - 5 } & & 1 & 2 & 3 \\
\hline P-CTR & 25 & 1281.40 & & 1281.40 \\
\hline T-CTR & 25 & & 3279.80 & 3279.80 \\
\hline PPROM & 50 & 1604.20 & 604.20 & \\
\hline Significance & & 0.641 & 1.000 & 1.000 \\
\hline
\end{tabular}

Sonographic estimated fetal weight (SEFW) in PPROM group (mean \pm SD: $1604.2+371$ ) Vs P-CTR group (mean \pm SD: $1281.4 \pm 599$ ) revealed that there was no significant difference between the two groups with post hoc significance $=0.641$ and $p>0.05$. Yet there was a significant difference between PPROM group and TCTR group (mean \pm SD: $3279.8 \pm 18$ ) with post hoc significance $=1$ and $\mathrm{p}<0.001$ (Table 4).

Table (5): The post-hoc Tukey test showing the difference in AFI between PPROM and Both P-CTR and TCTR groups.

\begin{tabular}{|l|c|c|c|c|}
\hline Groups & \multirow{4}{*}{$N$} & \multicolumn{3}{|c|}{ Subset for alpha= 0.05} \\
\cline { 3 - 5 } & $N$ & 1 & 2 & 3 \\
\hline$P$-CTR & 25 & 15.20 & & 15.20 \\
\hline T-CTR & 25 & & 13.85 & 13.85 \\
\hline PPROM & 50 & 3.56 & 3.56 & \\
\hline Significance & & 1.000 & 1.000 & 0.874 \\
\hline
\end{tabular}

PPROM group had an obvious significantly lower AFI (mean \pm SD: $3.56 \pm 3 \mathrm{~cm}$ ) compared with both P-CTR group (mean $\pm \mathrm{SD}$ ): $15.2 \pm 3.4 \mathrm{~cm}$ ) and T-CTR group (mean $\pm \mathrm{SD}: 13.8 \pm 5.8 \mathrm{~cm}$ ) with post hoc-significance $=1$ and $\mathrm{p}<0.001$. There were no significant difference in AFI between P-CTR group and T-CTR group with post hoc significance $=0.874$ and $p>0.05$. (Table 5).

Table (6): The mode of delivery in PPROM group

\begin{tabular}{|l|l|l|}
\hline & Frequency & Percent \\
\hline VD & 40 & 80.0 \\
\hline CS & 10 & 20.0 \\
\hline
\end{tabular}

Table (7): The fetal outcome in PPROM group

\begin{tabular}{|l|l|l|}
\hline & Frequency & Percent \\
\hline Still birth & 2 & 4.0 \\
\hline Living & 48 & 96.0 \\
\hline
\end{tabular}

$20 \%$ of PPROM women were delivered by $\mathrm{C} / \mathrm{S}$ (table 6) and the percentage of stillbirth was $4 \%$ (table 7).

One of the two stillbirths was case number 32 died in utero from hyperstimulation. While the other was case number 46 died due to failed neonatal resuscitation as the mother had a previable PPROM

\section{Sonographic estimated myometrial thickness}

Anterior wall, fundal, posterior wall and LUS myometrial thickness were compared within each group and each MT was compared among the three groups as follow:

\section{(1) In each group separately: 1 - The PPROM group:}

MT assessment in PPROM group revealed uniform thickness at each site of uterine body. The mean \pm SD in millimeters were the following: anterior wall $(9.9 \pm 1.8 \mathrm{~mm})$, fundal wall $(10 \pm 2.1 \mathrm{~mm})$, posterior wall $(9.0 \pm 1.6 \mathrm{~mm})$, [p=0.07].

However the LUS $(7.6 \pm 1.7 \mathrm{~mm})$ was significantly thinner in PPROM women compared with other sites $[\mathrm{p}<0.001]$.

Table (8): Myometrial thickness in the anterior wall, fundus, posterior wall and LUS in PPROM groups

\begin{tabular}{|l|l|}
\hline \multicolumn{1}{|c|}{ Uterine wall } & MT in mm (Mean \pm SD) \\
\hline Anterior wall & $9.9 \pm 1.8$ \\
\hline Fundus & $10 \pm 2.1$ \\
\hline Posterior wall & $9.0 \pm 1.6$ \\
\hline LUS & $7.6+1.7$ \\
\hline
\end{tabular}




\section{2-The T-CTR group:}

Sonographic evaluation of myometrial thickness (MT) at term (T-CTR) demonstrated that MT for each woman was uniform between uterine body sites. The mean \pm SD were the following: anterior wall $(8.8 \pm 0.3 \mathrm{~mm})$, fundal wall $(8.7 \pm 0.2 \mathrm{~mm})$, and posterior wall $(8.2 \pm 0.2 \mathrm{~mm})$. At term, all uterine body sites were significantly thicker than LUS: $(4.6 \pm 0.2 \mathrm{~mm})[\mathrm{p}<0.001]$.

\section{3-The P-CTR group}

In P-CTR group, the difference in MT between sites including the LUS did not reach statistical significance (mean $\pm \mathrm{SD}$ ): anterior wall $(7.2 \pm 0.2 \mathrm{~mm})$, fundal wall $(7.5 \pm 0.2 \mathrm{~mm})$, posterior wall $(7.3 \pm 0.3 \mathrm{~mm})$ and LUS $(6.3 \pm 0.1 \mathrm{~mm})[\mathrm{p}>$ $0.05]$.
Figure (1): Bar chart different uterine sites $($ mean \pm SD)

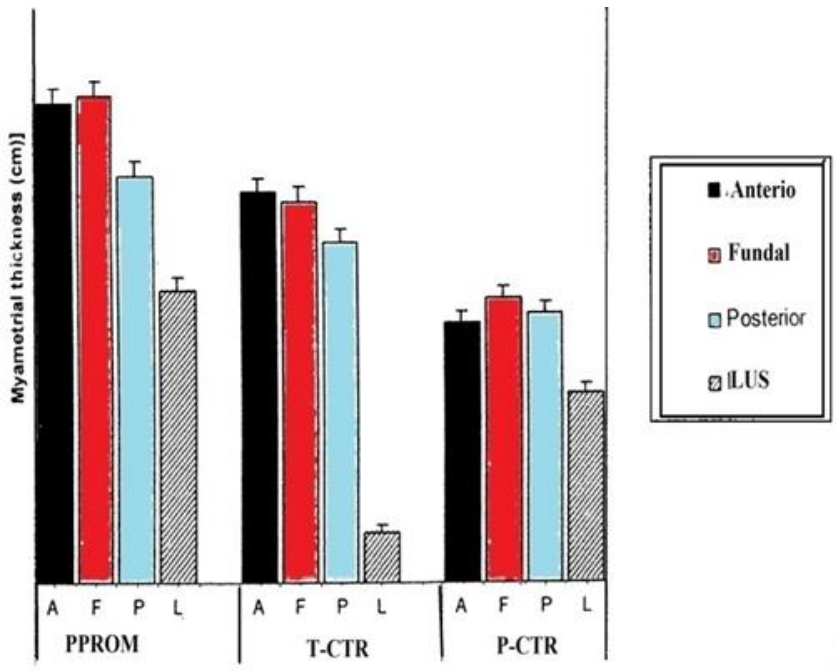

representing MT at in the three groups

\section{(2) Between each two groups separately}

\section{1 - Anterior wall:}

Table (9): The post-hoc Tukey test showing the difference in anterior wall MT between PPROM and both TCTR and P-CTR groups.

\begin{tabular}{|l|l|l|l|l|}
\hline Groups & N & \multicolumn{4}{|l|}{ Subset for alpha =0.05 } \\
\hline & & 1 & 2 & 3 \\
\hline P-CTR & 25 & 7.220 & & 7.220 \\
T-CTR & 25 & & 8.892 & 8.892 \\
PPROM & 50 & 9.910 & 9.910 & \\
Significance & & 1.000 & 1.000 & 1.000 \\
\hline
\end{tabular}

1. MT was significantly thicker at anterior site in PPROM group $(9.9 \pm 1.8 \mathrm{~mm})$ compared with P-CTR group $(7.2 \pm 0.2 \mathrm{~mm})$ with post hoc significance $=1$ and $\mathrm{p}<0.001$.

2. MT was also significantly thicker in PPROM group compared with T-CTR group $(8.8 \pm 0.3 \mathrm{~mm})$ with post hoc significance $=1$ and $\mathrm{p}<0.001$.

3. MT showed a significant difference between both P-CTR group and T-CTR group with post hoc significance $=1$ and $\mathrm{p}<0.001$ as the results are shown in Table (9). 


\section{2-Fundus:}

Table (10): The post-hoc Tukey test showing the-difference in fundal MT between PPROM and both P-CTR and T-CTR groups

\begin{tabular}{|l|l|l|l|l|}
\hline Groups & N & \multicolumn{3}{l|}{ Subset for alpha =0.05 } \\
\hline & & 1 & 2 & 3 \\
\hline P-CTR & 25 & 7.588 & & 7.588 \\
T-CTR & 25 & & 8.724 & 8.724 \\
PPROM & 50 & 10.012 & 10.012 & \\
Significance & & 1.000 & 1.000 & 0.871 \\
\hline
\end{tabular}

A. There was a significant difference in the fundal sites between PPROM group $(10.0 \pm 2.1 \mathrm{~mm})$ and PCTR $(7.5 \pm 0.2 \mathrm{~mm})$ with post hoc significance $=1$ and $\mathrm{p}<0.001$.

B. A significant difference was also maintained between PPROM group and T-CTR group $(8.7 \pm 0.2 \mathrm{~mm})$ with post hoc significance $=1$ and $\mathrm{p}<0.001$.

C. There was no significant difference between both P-CTR group and T-CTR group with post hoc significance $=0.871$ and $p>0.05$. The results are shown in table (10).

\section{3- Posterior wall:}

Table (11): The post-hoc Tukey test showing the difference in posterior wall MT between PPROM and both P-CTR and T-CTR groups

\begin{tabular}{|l|l|l|l|l|}
\hline \multicolumn{1}{|c|}{ Groups } & N & \multicolumn{4}{|l|}{ Subset for alpha =0.05 } \\
\hline & & 1 & 2 & 3 \\
\hline P-CTR & 25 & 7.388 & & 7.388 \\
T-CTR & 25 & & 8.292 & 8.292 \\
PPROM & 50 & 9.008 & 9.008 & \\
Significance & & 1.000 & 1.000 & 0.745 \\
\hline
\end{tabular}

a) There was a significant difference in MT of posterior wall in both PPROM group $(9.0 \pm 1.6 \mathrm{~mm})$ and TCTR group $(8.2 \pm 0.2 \mathrm{~mm})$ with post hoc significance $=1$ and $\mathrm{p}<0.001$.

b) There was a statistically significant difference of posterior wall thickness between PPROM group and P-CTR group $(7.3 \pm 0.3 \mathrm{~mm})$ with post hoc significance $=1$ and $\mathrm{p}<0.001$.

c) There was no significant difference between P-CTR group and T-CTR group with post hoc significance $=0.745$ and $\mathrm{P}>0.05$ as the results are shown in table (11).

\section{4-LUS:}

Table (12):The post-hoc Tukey test showing the difference in LUS MT between PPROM and each P-CTR and T-CTR groups

\begin{tabular}{|l|l|l|l|l|}
\hline \multicolumn{1}{|c|}{ Groups } & N & \multicolumn{3}{l|}{ Subset for alpha $=0.05$} \\
\hline & & 1 & 2 & 3 \\
\hline P-CTR & 25 & 6.352 & & 6.352 \\
T-CTR & 25 & & 4.640 & 4.640 \\
PPROM & 50 & 7.676 & 7.676 & \\
Significance & & 1.000 & 1.000 & 1.000 \\
\hline
\end{tabular}

a) The MT at LUS was significantly thicker in PPROM group $(7.6 \pm 1.7 \mathrm{~mm})$ compared with T-CTR group $(4.6 \pm 0.2 \mathrm{~mm})(\mathrm{p}<0.001)$.

b) There was also a highly significant difference between PPROM group and P-CTR group $(6.3 \pm 0.1 \mathrm{~mm})$ $(\mathrm{p}<0.001)$.

c) The difference was maintained between P-CTR group and TCTR group with $\mathrm{p}<0.001$. The results are shown in table (12). 


\section{(3)- In PPROM group:}

By using the univariant linear regression analysis, we modeled the latency interval as a dependent variable and MT (fundal. anterior wall, posterior wall and LUS), gestational age, AFI and fetal weight as independent variables to find out the strongest positive correlation between the latency interval and the independent variables.

Table (13): The Pearson correlation and $\mathrm{P}$ value between the latency interval and the independent variables

\begin{tabular}{|l|l|l|c|c|c|c|c|l|}
\hline & & Fundus & anterior & posterior & & Gestational & AFI & $\begin{array}{l}\text { Fetal } \\
\text { Weight } \\
(\mathrm{G})\end{array}$ \\
\hline $\begin{array}{l}\text { Latency } \\
\text { Interval } \\
\text { (hours) }\end{array}$ & $\begin{array}{l}\text { Pearson } \\
\text { correlation }\end{array}$ & $0.895(* *)$ & $0.866(* *)$ & $0.868(* *)$ & $0.813(* *)$ & $0.769(* *)$ & $0.710(* *)$ & 0.074 \\
\cline { 2 - 9 } & wig. (2-tailed) & 0.000 & 0.000 & 0.000 & 0.000 & 0.000 & 0.000 & 0.608 \\
\hline
\end{tabular}

$\begin{array}{cc}\text { Pearson correlation: } 0.3-0.5 & \text { moderate +ve correlation } \\ 0.5-0.7 & \text { strong +ve correlation } \\ >0.7 & \text { very strong +ve correlation, } \\ (* *) & \text { very strong pearson correlation }\end{array}$

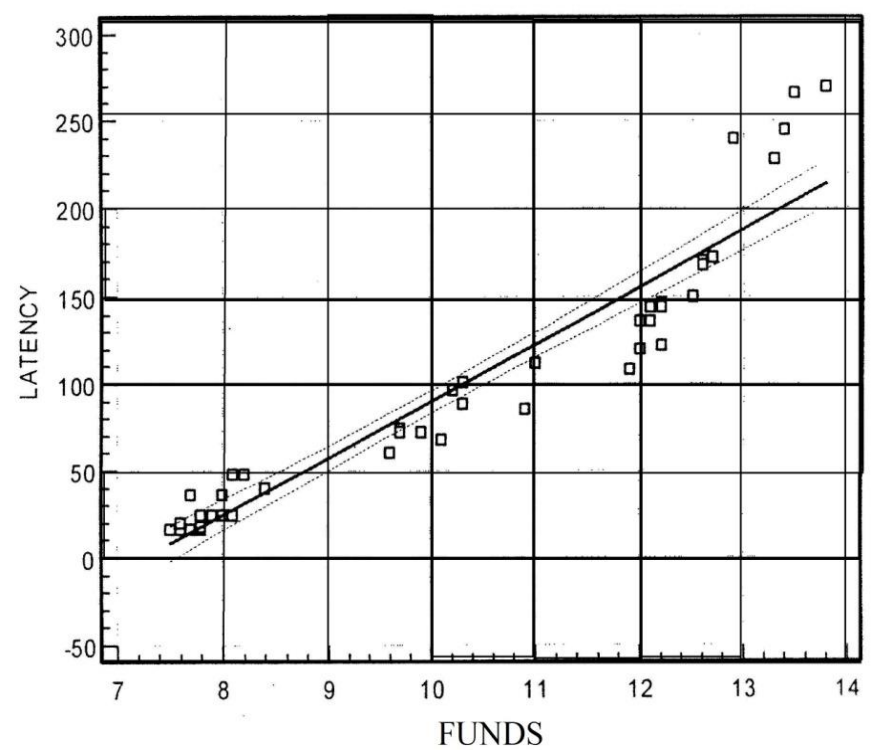

1-Latency interval and fundal MT

Figure (2): A scatter diagram showing the relation between fundal MT and latency interval in women with PPROM

Regression analysis suggested that there was a direct correlation between latency interval and fundal MT with a very strong +ve Pearson correlation $=0.895$ and a highly significant $p$ value $<0.001$ as shown in figure (2).

Latency interval $=($ fundal MT $x$ 32.7) -237.5

2-Latency interval and anterior wall MT: There was a direct +ve correlation between the latency interval and anterior MT with pearson correlation $=0.866$ and $\mathrm{p}<0.001$.

3-Latency interval and posterior wall MT: Posterior wall is positively correlated to the latency interval with pearson correlation $=0.868$ and $\mathrm{p}$ $<0.001$.

4-Latency interval and LUS MT: LUS is positively correlated to the latency interval with pearstm correlation $=0.813$ and $p<0.00 I$. From above correlation between latency interval and each wall MT, we found that fundal MT is the strongest independent variable correlated to the latency interval as it has the highest +ve pearson correlation.

5-Latency interval and AFI: There was a direct correlation between latency interval and AFI with pearson correlation $=0.710$ and $\mathrm{P}<0.001$. This means that the more the increase in the AFI the more the increase in time of the latency interval. 6-Latency interval and gestational age: There was a +ve pearson correlation between the latency interval and GA with pearson correlation $=0.769$ and $\mathrm{p} 0.001$. This means that the more the increase in the gestational ages the more the increase in the latency interval.

\section{DISCUSSION}

Spontaneous rupture of membranes is a normal component of labor and delivery. Rupture of membranes before the onset of labor is considered premature (PROM), and induction of labor is common if the patient is at or close to term. Patient 
management becomes more challenging when

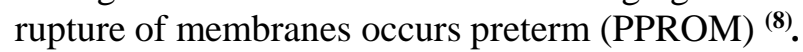

In the current study, we studied 100 pregnant women: PPROM $(\mathrm{n}=50)$, term non labor control ( $\mathrm{T}-\mathrm{CTR}, \mathrm{n}=25)$, and preterm non labor control (P-CTR, $n=25)$.

Conservative management of PPROM women was done by follow up including temperature chart every 6 hours, total leucocytic count (TLC) and C-reactive protein (CRP) twice weekly as well as assessment of fetal wellbeing.

Maternal age, maternal weight, number of previous pregnancies and gestational age were considered as demographic and clinical variables, while SEFW, AF1 and MT were assessed as sonographic variables.

As regarding the maternal weight, there was no significant difference among the three groups $(p>0.05)$ as shown in table $(1,2)$. Thus we avoided the misleading results in ultrasound assessment which are common in obese women due to thick anterior abdominal wall ${ }^{(\mathbf{1 4})}$.

Our results revealed that there was no significant difference in number of previous pregnancies among the three groups $(p>0.05)$ as shown in tables $(1,2)$. Thus, obviating any influence of the number of previous pregnancies on the MT. This is due to the fact that the more the increase in number of previous pregnancies, the more the permanent thinning of the myometrium ${ }^{(14)}$. Thus, it would be not realistic to compare MT in multiparous women and primigravida.

There was no significant difference in gestational age between PPROM and P-CTR group. This enabled us to compare MT between the two groups in the same gestational age range.

SEFW showed no significant difference between PPROM and P-CTR groups $(p>0.05)$ as shown in table (4). This is because the two groups were in the same gestational age.

In the present study, MT was sonographically measured in 4 different sites in the three groups.

MT assessment in the PPROM group revealed uniform thickness at each site of uterine body (anterior wall, fundal wall, posterior wall) $(p>0.05)$. However the LUS was thinner in PPROM women compared with other sites $(\mathrm{p}<0.001)$ as shown in table (8) and figure (1).

The same results were obtained by Kalantari et $\boldsymbol{a l} .{ }^{(18)}$, who had made a cross-sectional study on 24 cases with PPROM, measuring the myometrial thickness in them as an applicable and noninvasive method in predicting the length of latency interval of labor (the period from PPROM to start of labor).

In our study sonographic evaluation of myometrial thickness (MT) at term (T-CTR) demonstrated that MT for each woman was uniform between uterine body sites anterior wall, fundal wall, posterior wall $(p>0.05)$. All uterine body sites were significantly thicker than the lower uterine segment (LUS) $(\mathrm{p}<0.001)$.

The same results were obtained by Kalantari et al. ${ }^{\left({ }^{18)}\right.}$ and Buhimschi et al. ${ }^{\left({ }^{(8)}\right.}$ they demonstrated that MT was uniform between uterine body sites anterior wall: $8.8 \pm 0.5 \mathrm{~mm}$, fundal: $8.6 \pm 0.4 \mathrm{~mm}$, posterior wall: $8.2 \pm 0.3 \mathrm{~mm}$ ( $\mathrm{p}>0.05)$. At term; all uterine body sites were thicker than the LUS: $4.7 \pm 0.5 \mathrm{~mm} \quad(\mathrm{p}<0.001)$. As term patients were approaching labor, marked thinning of LUS seems to be an appropriate explanation for the above mentioned results.

In P-CTR group, the difference in MT between sites including the LUS did not reach statistical significance $(p>0.05)$ (figure, 1$)$. These results agreed with the results of Kalantari et al. (18), Buhimschi et al. ${ }^{\left({ }^{(8)}\right.}$ which revealed that in the PCTR group the difference in MT between sites including the LUS did not reach statistical significance; anterior: $7.2 \pm 0.4 \mathrm{~mm}$, fundal: $7.7 \pm 0.4 \mathrm{~mm}$, posterior: $7.4 \pm 0.4 \mathrm{~mm}$, LUS: $6.2 \pm 0.5 \mathrm{~mm} \quad(p>0.05)$. This uniform thickness among sites released the fact that the LUS were not yet formed.

Sonographic evaluation revealed that MT was significantly thicker at the anterior site in PPROM group compared with both P-CTR group and TCTR group $(\mathrm{p}<0.001)$ (table 9). This difference was maintained in the fundal site in the PPROM group compared with both P-CTR group and T-CTR group $(\mathrm{p}<0.001)$ (table 10). This difference was also maintained in the posterior wall where there was a significant difference between PPROM groups and both P-CTR and T-CTR groups $(\mathrm{p}<0.001)$ (table 11). These results were similar to the results of Buhimschi et al. ${ }^{(8)}$ which revealed the same differences among groups with $\mathrm{p}<0.001$.

It seems to be reasonable to conclude that the event of PPROM led to a diminished uterine wall distention due to the sudden decompression of the uterine sac which had been filled with a minimally compressible fluid that was normally opposing thickening provided that there is no contractile activity present Kalantari $\boldsymbol{e t ~ a l .}{ }^{(18)}$.

The LUS was significantly thicker in PPROM group compared with both T-CTR and PCTR groups $(p<0.001)$. This difference was maintained between P-CTR and T-CTR groups (table 12).

These results agreed with the results of Kalantari et al. ${ }^{(18)}$ and Buhimschi et al. ${ }^{(8)}$ which revealed that the LUS was significantly thicker in PPROM than in both P-CTR and T-CTR groups. 
The diminished uterine wall stress following PPROM was the most acceptable explanation for the thicker LUS, while the stage of myometrial activation in T-CTR groups explained why the LUS was significantly thinner compared with the LUS in P-CTR groups $(\mathrm{p}<0.001)$.

Regression analysis suggested that there was a very strong positive person correlation $=0.895$ between the latency interval and the fundal myometrial thickness and the $\mathrm{p}$ value was highly significant ( $\mathrm{p}<0.001)$. (table 13).

By using the linear regression model, we modeled the latency interval as a dependant variable and the fundal myometrial thickness as a predictor (constant), thus an equation was formed to calculate the period of the latency interval from the sonographically estimated fundal myometrial thickness (figure 2).

\section{Latency interval $=($ fundal MT $x$ 32.7) -237.5}

Our results revealed also there was a direct positive Pearson correlation between the latency interval and both the anterior wall and the posterior wall with Pearson correlation $=0.866$ and 0.868 respectively. Also the LUS is correlated to the latency interval with Pearson correlation $=0.813$.

In spite of different statistical analysis used, Buhimschi et al. ${ }^{(8)}$ found the strongest correlation between the fundal MT and the latency interval. They listed the sensitivities and specificities of fundal MT measurement for the prediction of the latency interval with the optimum cut-offs in predicting delivery from 48 to 168 hours. MT less than $12.1 \mathrm{~mm}$ was $93.7 \%$ sensitive and $63.6 \%$ specific for the identification of women whose latency period was less than 120 hours.

We preferred to use the linear regression model to calculate the coefficient of the latency interval. This was due to the fear that to detect sensitivity and specificity of the MT, we had to identify a cutoff value for the latency interval below and above which the results of MT estimated could be assumed.

Our regression analysis model is strongly fit for $95 \%$ of women with PPROM with fundal MT above $7 \mathrm{~mm}$ although those women among the confidence interval (CI) with fundal MT above $12 \mathrm{~mm}$ were not strongly fit for the model. Otherwise, the results of Buhimschi et al. (8) estimated a cut-off value for the fundal MT = $12.1 \mathrm{~mm}$ above which latency interval was more than 120 hours and below which latency interval was less than 120 hours.

The study of, Kalantari et al. ${ }^{(18)}$ and our study assumed that women with a long latency interval after spontaneous PPROM are in a state of myometrial quiescence or incomplete myometrial activation, and demonstrate that the long latency and presumed myometrial quiescence are associated with greater thickness of the anterior and fundal wall myometrium.

Our results revealed that the mean $\pm \mathrm{SD}$ of AFI in the PPROM group was $3.56 \pm 3.1 \mathrm{~cm}$. By using the linear regression analysis, we detected a strong positive correlation between the AFI and the latency interval with Pearson correlation $=0.710, \mathrm{p}<0.001$.

Similar results were obtained by Park et al. ${ }^{(19)}$. They studied the relation between oligohydramnios and the onset of preterm labor in PPROM. Their results revealed that spontaneous preterm delivery within 24 hours and 48 hours was more frequent among patients with an amniotic fluid index of $\leq 5$ $\mathrm{cm}$ than those with an amniotic fluid index of $>5$ $\mathrm{cm}$. Moreover, Cox proportional hazards model analysis indicated that an amniotic fluid index of $\leq 5$ $\mathrm{cm}$ was a significant predictor of the duration of the pregnancy after adjustment for gestational age. The mean \pm SD of gestational age in PPROM group was $30.7 \pm 2.8$ weeks and by using the linear regression analysis, we found a positive Pearson correlation $=0.769$ between the gestational age and the latency interval with $\mathrm{p}<0.001$. These results agreed with the results of Fuks et al. ${ }^{(20)}$ who studied 249 women at different gestational ages at the time of PPROM. Their results revealed that there was a strong Pearson correlation $=0.834$ between the gestational age and the latency interval.

\section{CONCLUSION}

From the previous study, it could be concluded that there was a significant thickening of the fundal walls of the uterus follow PPROM. A thick myometrium in no laboring PPROM women is associated with long latency interval.

\section{RECOMMENDATIONS}

Conservative management of PPROM especially in developing countries is mandatory to overcome the neonatal morbidity and mortality and where there are no highly equipped neonatal centers.

Prediction of the latency interval remains an obscure confine in the art of obstetrics.

Estimation of the myometrial thickness as a predictor for the latency period in case of PPROM seems to have an accepted accuracy.

However, viewed, the large number of coexisting variables and the possibility of intra and inter-observer variability, it should be integrated as a part of multiple tools, namely AFI, gestational age, fetal fibronectin and assessment of cervical length and/or index. 
Assessment of the posterior uterine wall MT is the least accurate compared to the other uterine walls. Fundal and anterior wall MT measurements are both easier to be measured and more positively correlated to the latency interval.

Several factors may affect women with PPROM during the latency interval like intraamniotic infection. Thus it is suggested to measure the MT periodically every three or four days for accurate estimation of the latency interval.

\section{REFERENCES}

1. Kumar D, Moore RM, Mercer BM et al. (2016): The physiology of fetal membrane weakening and rupture: Insights gained from the determination of physical properties revisited. Placenta, 42: 59-73.

2. Begum $\mathbf{R}$ (2018): Premature Birth. Patient Management in Obstetrics and Gynecology. Am $\mathbf{J}$ Obstet Gynecol., 61(1); 120-9.

3. Mercer BM (2003): Preterm premature rupture of membranes. Obstet Gynecol., 101 ;178-93.

4. Dorfeuille N, Morin V, Tétu A et al. (2016): Vaginal fluid inflammatory biomarkers and the risk of adverse neonatal outcomes in women with PPROM. American Journal of Obstetrics and Gynecology, 214(1): 290-295.

5. Friedman P, Ogunyemi D (2018): Oligohydramnios. In Obstetric Imaging: Fetal Diagnosis and Care (Second Edition), https://www.elsevier.ca/ca/product.jsp?isbn=97803 23497367

6. Waldorf KM, Singh N, Mohan AR et al. (2015): Uterine overdistention induces preterm labor mediated by inflammation: observations in pregnant women and nonhuman primates. American Journal of Obstetrics and Gynecology, 213(6): 830-831.

7. Anderson HF (2000): Use of fetal fibronectin in women at risk for preterm delivery. Clin Obstet Gynecol., 43(4);746-58.

8. Buhimschi IA, Christner $R$ and Buhimschi CS (2005): Proteomic biomarker analysis of amniotic fluid for identification of intra-amniotic inflammation. BJOG., 112; 173-81.

9. Ashwal E, Aviram A, Wertheimer A et al. (2016): The impact of obstetric gel on the second stage of labor and perineal integrity: a randomized controlled trial. The Journal of Maternal-Fetal and Neonatal Medicine, 29(18): 3024-3029.

10. Elshikh WA (2005): Sonographic evaluation of Myometrial Thickness as a Prognosticator for the Latency Interval in Pregnant Women with Preterm Premature Rupture of Membranes and Oligohydramnios. Am J Obstet Gynecol., 193(3 Pt 1):762-70.

11. Lams JD, Goldenberg RL, Meis PJ et al. (2003): The length of the cervix and the risk of spontaneous premature delivery. N Engle J Med., 334:567-72.

12. Weish A, Nicolaides $K$ (2002): Cervical screening for preterm delivery .Curr Opin Obstet Gynecol., 14: 195-202.

13. Bergelin I, Valentin L (2001): Patterns of normal change in cervical length and width during pregnancy in nulliparous women: longitudinal ultrasound. Ultrasound Obstet Gynecol., 18:217-22.

14. Degani S, Leibovitz I, Shapiro R et al. (1998): Myometrial thickness in pregnancy: longitudinal sonographic study. J Ultrasound Med., 17(10):6615.

15. Xiao ZH, Andre $P$, Lacaze-Masmonteil $T$ et al. (2000): Outcome of premature infants delivered after prolonged premature rupture of membranes before 25 weeks of gestation. Euro J Obstet Gynecol Report Biol., 90(1): 67-71.

16. Phelan JP, Smith CV, Broussard P et al. (1987): Amniotic fluid volume assessment with the fourquadrant technique at 36-42 weeks' gestation. J Report Med., 1987: 32(7):540-2.

17. Hadlock FP, Harrist RB, carpenter RJ et al. (2004): Sonographic estimation of fetal weight: the value of femur length in addition to head and abdomen measurement. Radiology, 150:535538.

18. Kalantari N, Khoshnood M, Shariati M et al. (2012): Correlation Between Myometrial Thickness and the Latency Interval in Preterm Premature Rupture of Membranes .Iran J Radiology, 7(4)215219.

19. Park JS, Yoon BH, Romero R et al. (2001): The relationship between oligohydramnios and the onset of preterm labor in preterm premature rupture of membranes. Am J Obstet Gynecol., 184(3): 459-62.

20. Fuks AM, Rajjendran G, Netta D et al. (2002): 249 gestational age at the time of preterm premature rupture of membranes of parturition. Am J Obstet Gynecol., 178: 6-15. 